\title{
Microchip Electrode Development for Traveling wave Dielectrophoresis of Non-Spherical Cell Suspensions*
}

\author{
Sakshin Bunthawin ${ }^{1 \#}$, Jatuporn Kongklaew ${ }^{1}$, Adisorn Tuantranont ${ }^{2}$, Kata Jaruwongrangsri ${ }^{2}$, Thitima Maturos $^{2}$ \\ ${ }^{1}$ Biotechnology of Electromechanics Research Unit, Science of Physics, Faculty of Technology and Environment, \\ Prince of Songkla University, Kathu, Phuket, 83120 Thailand \\ ${ }^{2}$ Nano-Electronics and MEMs laboratory, National Electronics and Computer Technology Center, \\ Pathumthani, 12120 Thailand \\ Email: "Sakshin.b@phuket.psu.ac.th
}

Received 2012

\begin{abstract}
A microchip interdigitated electrode with a sequential signal generator has been developed for traveling wave dielectrophoresis (twDEP) of biological cell suspensions. The electrode was fabricated on a microscope glass slide and coated with a $0.5 \mu \mathrm{m}$ thickness of gold through a sputtering technique which was designed for large-scale inductions of cells rather than for individual cells as in previous versions of our device. As designed for a representative cell size of $10 \mu \mathrm{m}$, the electrode array was $50 \mu \mathrm{m}$ in width to allow large numbers $\left(>10^{6}\right)$ of cells to be processed. The sequential signal generator produces an arbitrary AC quadrature-phase to generate traveling electric field for a microchip interdigitated electrode. Each phase signal can be automatically altered and alternated with the other phases within interval time of 0.01-30 seconds (controlled by programming). We demonstrate the system could be used to estimate the dielectric properties of the yeast Saccharomyces cerivisiae TISTR 5088, the green alga Tetraselmis sp. and human red blood cells (HRBCs) through curve-fitting of dielectro- phoretic velocities and critical frequencies.
\end{abstract}

Keywords: Traveling wave Dielectrophoresis; Microchip Interdigitated Electrode; Cell Suspensions; Dielectric Properties

\section{Introduction}

It is well known that a micro-interdigitated electrode can be employed for dielectrophoresis (DEP) and traveling wave dielectrophoresis (twDEP) of cell suspensions [1-4]. The microelectrodes for such work can generally be classified as three types: a one planar linear interdigitated array [1,5-7], two parallel arrays [2-4,8,9] and a multi-concentric ring structure [10]. Such systems have considerable biotech- nological potential. It is important to develop a user friendly version of the technology that is able to be used routinely in a biotechnology laboratory on a variety of types of cells.

The two-parallel electrode array might offer advantages for both of cell manipulation and separation. The methods allow cell suspensions to move either toward the electrode tips or along the electrode track simultaneously, as a result of two driving orthogonal forces [11]. Wang et al. [4] were the first to term them the "unified force" for a dielectric sphere which depends on both the real ( $\mathrm{Re}[\mathrm{CMF}])$ and the imaginary part (Im[CMF]) of the complex dielectric-frequency dependent Clausius-Mossotti factor [CMF]. The model was then extended for a shelled spheroidal dielectric by [11]. These analyses describe cell behavior in a traveling electric field and the models can be used to evaluate cell dielectric properties through curve-fitting of experimentally measured dielectrophoretic velocities and two critical frequencies.

The present study proposes a microchip interdigitated elec-

${ }^{*}$ Faculty of Technology and Environment and Prince of Songkla University are sponsors for the trip to BEB 2012.

${ }^{\#}$ Corresponding author. trode of two parallel arrays equipped with a sequential signal generator for experiments on traveling wave dielectrophoresis (twDEP) of cell suspensions. This microelectrode is designed for large-scale inductions of cells rather than for individual cells in our previous versions of the device such as "the octa-pair interdigitated electrode” described by [11]. The sequential signal generator generates traveling electric field of an arbitrary AC quadrature-phase to apply for type of cell. Dielectrophoresis and traveling wave dielectrophoresis spectra of a non-spherical unicellular green alga, Tetraselmis sp., yeast of Saccharomyces cervisiate TISTR 5088 and human red blood cells (HRBCs) were investigated. A curve-fitting method of cell velocities and the lower critical frequency (LCF) were employed to determine their dielectric properties using a spheroidal cell model proposed in our previous work. Our newly developed system is a significant step towards biotechnology applications of the technology.

\section{Theoretical Approaches}

\subsection{Traveling Dielectrophoretic Force}

In AC electric field, the time-average dielectrophoretic force $\left(\overrightarrow{\vec{F}}_{t}\right)$ [4] acting on a shelled spheroidal dielectric possessing the volume $4 \pi a b^{2} / 3$ (Figure 1) in a non-uniform sinusoidal traveling electric field of magnitude $E$ is given by [11]

$$
\overline{\vec{F}}_{t}=\frac{2}{3} \pi a b^{2} \varepsilon_{0} \varepsilon_{s}\left[\begin{array}{l}
\operatorname{Re}[\mathrm{CMF}] \nabla E^{2}+\operatorname{Im}[\mathrm{CMF}] \\
\left(E_{x}^{2} \nabla \varphi_{x}+E_{y}^{2} \nabla \varphi_{y}+E_{z}^{2} \nabla \varphi_{z}\right)
\end{array}\right]
$$

where $\mathrm{Re}[\mathrm{CMF}]$ and $\operatorname{Im}[\mathrm{CMF}]$ are the real and the imaginary 
parts of the complex dielectric- frequency dependent Clausius-Mossotti factor [CMF], $\varepsilon_{0}$ and $\varepsilon_{\mathrm{s}}$ are dielectric constants of the vacuum and the suspending medium, respectively.

The value of $E$ and its three components in the Cartesian co-ordinate frame $x, y, z \quad\left(E_{x}, E_{y}, E_{z}\right)$ is the root mean square (rms) and $\phi$ is the electrical phase sequences addressing onto each tip of the microelectrode (Figure 2). The real part of the time-average dielectrophoretic force $\left(\vec{F}_{t}\right)$ represents the conventional dielectrophoretic force $\left(\vec{F}_{C D E P}\right)$ and the imaginary term is the traveling wave dielectrophoretic force $\left(\vec{F}_{\text {twDEP }}\right)$.

\subsection{The Clausius-Mossotti Factor (CMF)}

The Clausius-Mossotti factor (CMF) of a shelled spheroidal model [11] possesses the physical parameters of the shell (cell membrane) thickness $(\delta)$ and complex dielectric constants of the shell $\left(\varepsilon_{m}^{*}\right)$, the cytoplasm $\left(\varepsilon_{c}^{*}\right)$ and the suspending medium $\left(\varepsilon_{s}^{*}\right)$, respectively. As for the whole spheroid, the dielectric properties can be considered as a single value of $\varepsilon_{\text {eff }}^{*}$, the so called "the effective value of the complex relative permittivity". The [CMF] factor is defined as [12],

$$
[\mathrm{CMF}]=\frac{\varepsilon_{\text {eff }}^{*}-\varepsilon_{s}^{*}}{\varepsilon_{s}^{*}+\left(\varepsilon_{e f f}^{*}-\varepsilon_{s}^{*}\right) L_{k}}
$$

As is seen from (2) $[11,13]$, both of Re[CMF] and Im[CMF] are affected by the dielectric and conductivity parameters of the cytoplasm $\left(\varepsilon_{c}, \sigma_{c}\right)$, the membrane $\left(\varepsilon_{m}, \sigma_{m}\right)$ and the suspending medium $\left(\varepsilon_{s}, \sigma_{s}\right)$. The values of the parameters chosen were of particular relevance to cells.

\subsection{Translational Velocity and Two Critical Frequencies}

Evaluation of cell translational velocity can be made using Newton's first laws of motion. For the present analysis, the electric field gradient was assumed to be so low that the trans- lational velocity of the spheroid will be essentially constant, that is, the particle has reached terminal velocity, where the translational force is balanced by the frictional drag force [11]. The translational speed of a spheroid for dielectrophoresis (DEP) and traveling wave dielectrophoresis (twDEP) are,

$$
\begin{gathered}
v_{D E P}=\frac{\varepsilon_{0} \varepsilon_{s} b^{2} \operatorname{Re}[C M F] \nabla E^{2}}{9 \eta K^{\prime}}, \\
v_{t w D E P}=\frac{\varepsilon_{0} \varepsilon_{s} b^{2} \operatorname{Im}[C M F] \sum_{i=x, y, z}\left(E_{i}^{2} \nabla \varphi_{i}\right)}{9 \eta K^{\prime}} .
\end{gathered}
$$

where $\eta$ is the viscosity of the suspending medium and $K^{\prime}$ is a shape factor. The direction of $v_{C D E P}$ and $v_{t w D E P}$ depends on the polarity of the $\operatorname{Re}[\mathrm{CMF}]$ and $\operatorname{Im}[\mathrm{CMF}]$, respectively. Both velocities are written as a function of cell dielectric properties and they are frequency dependent. In case of the dielectrophoretic force, the spheroid experiences positive dielectrophoresis if the field frequency is in the range from the lower critical frequency $\left(f_{\ell}\right)$ to the higher critical value $\left(f_{h}\right)$. The higher critical frequency value is usually so high that it is not measurable experimentally [11].

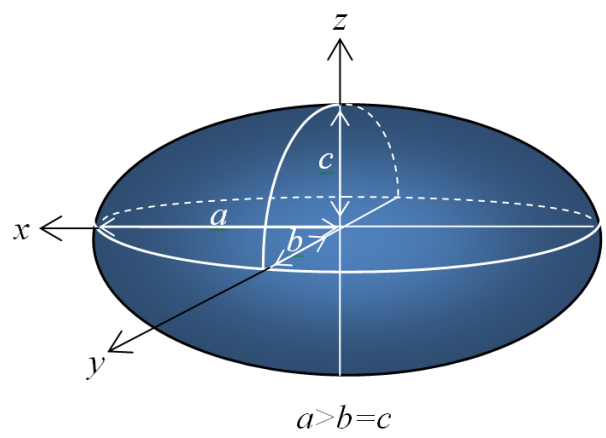

Figure 1. Prolate-spheroidal cell geometry in three orthogonal axes.

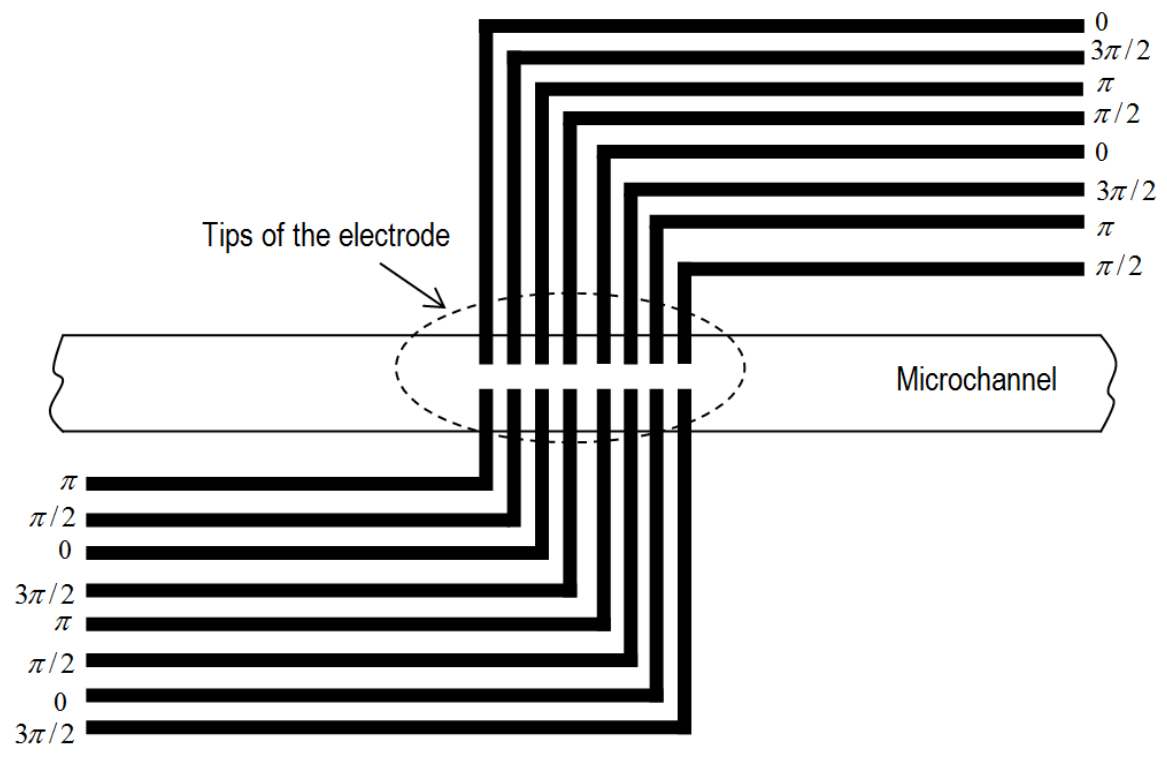

Figure 2. Diagram of the electrode configuration in top view. The track length of the microchannel is about $750 \mu \mathrm{m}$ and electric phase sequences (in radians) are addressed to the tips of the electrode as the quadrature pattern. 


\section{Experimental}

\subsection{Microelectrodes}

The present version of the octa-pairs interdigitated gold electrode was fabricated on microscope glass slides of dimension $75^{\times}{ }_{25} \times 1 \mathrm{~mm}$ (Marienfeld, Germany) (Figure 2). The electrode was strengthened by coating with a $0.5 \mu \mathrm{m}$ thickness of gold through a sputtering technique. Processes of photolithography and wet-etching were employed to finalize a prototype of the microelectrode. The electrode array was $50 \mu \mathrm{m}$ in width and the separation of the adjacent bars on the same array was $50 \mu \mathrm{m}$ and the microchannel width between the two arrays was $50 \mu \mathrm{m}$. The microchannel track length was about $750 \mu \mathrm{m}$. The glass slide of the microelectrode was mounted on the socket base shown in (Figure 3). An inferometer was employed to measure the thickness of the electrode tips.

\subsection{Electrical Setup}

The present version of the microchip interdigitated electrode equipped with the sequential quadrature-signal generator was developed from the previous version [11]. The sequential quadrature-signal generator was the source generating the traveling electric field of a quadrature phase for the 16 tips of the microchip octa-pair interdigitated electrode. The generator also has a function to split the electrical phase sequences $(\phi)$ i.e. $\phi=0, \pi / 2, \pi, 3 \pi / 2$ in series. They can be automatically be altered and circulated to be in or out of phase within interval times of $0.01-30$ seconds (controlled by programming). The equipment is connected directly to the microelectrode via the computer cables and the microchip junction unit [11].

\subsection{Cells Preparation}

Yeast cells of Saccharomyces cerivisiae TISTR 5088 were determined as a prolate spheroids of $a=4.0 \pm 0.7$ and $b=3.0 \pm$ $0.4 \mu \mathrm{m}$ (mean $\pm \mathrm{SD}$ ). They were cultured in Biotechnology of Electromechanics research unit, Prince of Songkla University. The cells were harvested in the stationary phase after $24 \mathrm{hr}$ and washed twice with deionized water, then centrifuged at 1,000× $\mathrm{g}$ for $2 \mathrm{~min}$ and re-suspended twice in $0.5 \mathrm{M}$ sorbitol solution. The solution conductivity $\left(\sigma_{s}\right)$ was measured by using a conductivity meter (Eutech Instruments, Cyberscan CON 11), the conductivity was adjusted to be in the range of 3 to 300 $\mathrm{mS} . \mathrm{m}^{-1}$ by adding $0.1 \mathrm{M} \mathrm{KCl}$ solution, using a micropipette (Nichipet, model 5000DG). For the experiments with dead yeast cells, the cell suspension was heated to $75^{\circ} \mathrm{C}$ for $10 \mathrm{~min}$ [8] and then cooled down to room temperature $[8,14]$. The suspension was then centrifuged as described for viable cells.

Phytoplankton of Tetraselmis sp., obtained from the National Institute for Coastal Aquaculture (NICA), Songkla, Thailand. The cells were cultured in Sato and Serikawa's artificial seawater and harvested when the cells were in log-growth phase. They were considered as a spheroid with average dimensions $a$ $=10.0 \pm 0.7 \mu \mathrm{m}$ and $b=8.0 \pm 0.5 \mu \mathrm{m}$. Centrifugations at 7,000 rpm for 2 min were made to re-suspend twice in $0.5 \mathrm{M}$ sorbitol solution as described by [14]. The solution conductivity was measured by using a conductivity meter (Tetracon 325, LF318), and it was adjusted to be between 3 and $300 \mathrm{mS} . \mathrm{m}^{-1}$ by adding $0.1 \mathrm{M} \mathrm{KCl}$ solution, using a micropipette (Nichipet, model $5000 \mathrm{DG})$. Non-viable cells were prepared by boiling the cells at $80{ }^{\circ} \mathrm{C}$ for $10 \mathrm{~min}$, cooling down to room temperature, centrifuging and re-suspending in the same experimental solution as was used for the live cells [14]. Arsenic pre-treated cells were prepared by adding arsenic solution (Sodium arsenite, $\mathrm{NaAsO}_{2}$, MW 129.9) into the cell culture at concentrations varying from 1 to $150 \mathrm{ppm}$ and leaving them for 24 hrs before being used in an experiment.

Human red blood cells (HRBCs) were obtained directly from the blood bank of Vachira Phuket Hospital (Phuket, Thailand). The normal mature red cells of blood group A and B were used in the present study. Cell were centrifuged at $1,000 \mathrm{~g}$ for $2 \mathrm{~min}$ then resuspended twice in isotonic sorbitol (300 mOsmol. $\left.\mathrm{kg}^{-1}\right)$. The solution conductivity $\left(\sigma_{s}\right)$ was adjusted to be between 3 to $300 \mathrm{mS} \cdot \mathrm{m}^{-1}$ by adding $0.1 \mathrm{M} \mathrm{KCl}$ solution. HRBCs were considered as an oblate spheroid with average radius dimensions $a=$ $3.5 \pm 0.3 \mu \mathrm{m}, b=3.0 \pm 0.5 \mu \mathrm{m}$ and $c \approx 1.5 \mu \mathrm{m}$.

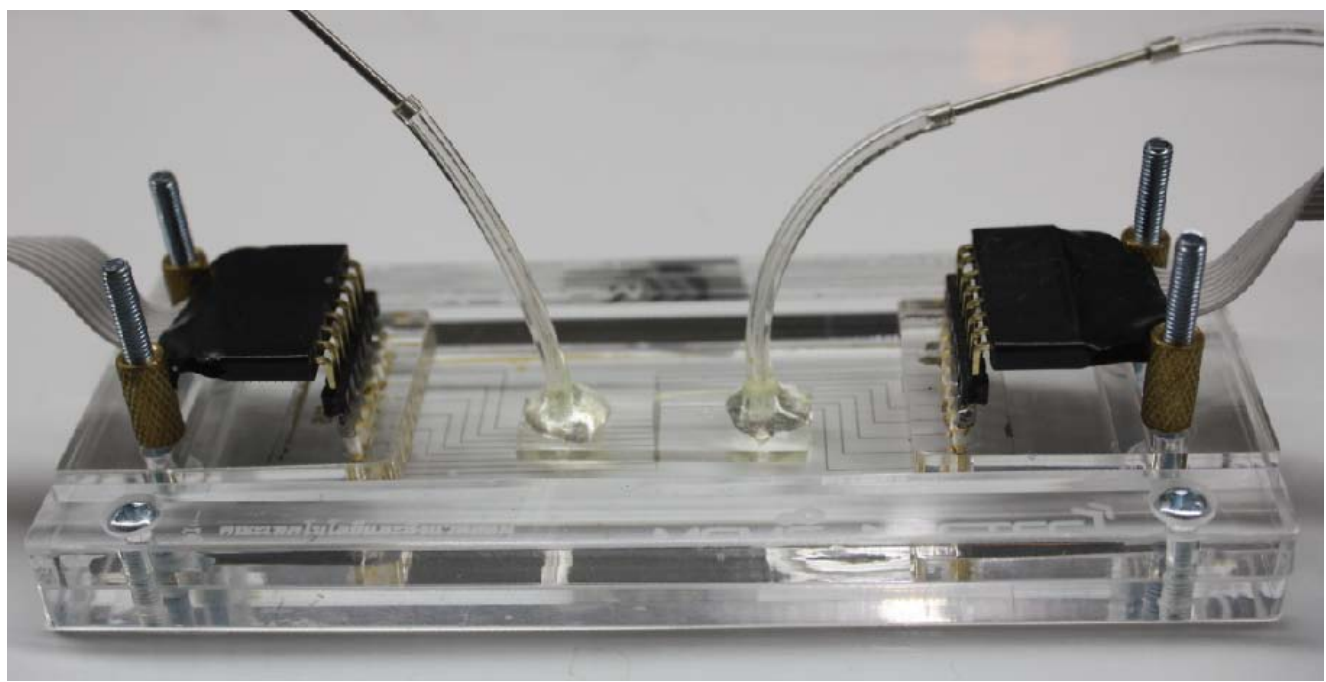

Figure 3. Microchip interdigitated electrode on the glass slide covered with PDMS sheet. The electrode has a fluid circulation system to flow cell suspensions through the microchannel of the electrode using microtubes connected at the end of the track. 


\subsection{Data Collection}

For the DEP measurements $100 \mathrm{ml}$ aliquots of a diluted cell suspension containing $10^{6}$ cells $/ \mathrm{ml}$ were pipetted onto the glass slide between the microelectrodes. The electrodes were energized with four sinusoidal signals (the quadrature phase) of amplitudes $0.7,1.4,2.8$ and $7.0 \mathrm{~V}$ (rms) in the phase sequence. To determine the lower critical frequency $\left(f_{\ell}\right)$, the frequency of the applied signals was gradually decreased from the upper value of $4 \mathrm{MHz}$ to the lower values (down to $5 \mathrm{kHz}$ ).

\subsection{Data Fitting}

Cells dielectric parameters were estimated through curve-fitting of dielectrophoretic velocities and lower critical frequencies. The method to fit the theoretical curves of cell velocity and the lower critical frequency with that of the experiments were made based on the known sensitivities of dielectric parameters [11].

\section{Results and Discussion}

\subsection{Microelectrodes}

A microchip of two array-interdigitated electrode equipped with a sequential signal generator was developed for a study of traveling wave dielectrophoresis (twDEP) of biological cells. This prototype was designed for large-scale inductions of cells suspensions rather than for individual cells in previous versions of the device. The track length was about $750 \mu \mathrm{m}$ for a microchannel, hence it allows large numbers $\left(>10^{6}\right.$ cells $\left./ \mathrm{ml}\right)$ of cells to be processed. The electrode tips were strengthened by coating with a $0.5 \mu \mathrm{m}$ thickness of gold which is thicker than that of the previous version. The sequential signal generator is designed for use on a laboratory scale and programmed to drive the quadrature signal for induction of the traveling electric field.

For the present study, the quadrature-phase difference ad- dressing on the adjacent and the opposite electrodes are of $\pi / 2$ and $\pi$, respectively. The phase sequence can automatically be altered and circulated within a interval time of 0.01-30 seconds. The operating frequency was ranged from $5 \mathrm{~Hz}$ to 4 $\mathrm{MHz}$ with the output voltage range of $1.5-14 \mathrm{~V}_{\mathrm{pp}}$. As simulated from the twDEP force model, the frequency dependent twDEP force acting on a biological cell can be resolved into two orthogonal forces which are determined by the real and the imaginary parts of the Clausius-Mossotti factor [CMF]. The former is determined by the gradient in the electric field and a $\pi$-difference pattern of the signals where the DEP force directs a cell either towards or away from the tip of the electrodes. The imaginary component is in a direction along the track of the electrode array which is determined by a signal of $\pi / 2$-difference. Simulations of twDEP force acting on a shelled dielectric allow one to obtain the optimization of phase sequences changes addressing on each bar of the electrode. Such simulations yield a better understanding of twDEP force and allow one to obtain the optimum phase sequence addressed to each bar of the electrode. For example of Tetraselmis, at 60 $\mathrm{kV} \cdot \mathrm{m}^{-1}, 300 \mathrm{kHz}$, and the conductivity of cell suspension me-

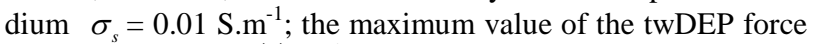
was found to be $20 \times 10^{-4} \mathrm{pN}$ which corresponds to a cell velocity of $2.4 \mu \mathrm{m} \cdot \mathrm{s}^{-1}$.

\subsection{Cell Inductions}

As was expected from previous experience with yeast, Tetraselmis sp. and human red blood cells (HRBCs), increasing electric field strengths resulted in increased values of cell velocity and an increase in the peak of the positive dielectrophoresis spectra (Figure 4). The velocity spectra of a cell were obtained by measuring cell velocity during its movement towards the electrode tip, under various field frequencies. Lower critical frequency ( $f_{\ell}$ ), where the cell was repelled (i.e. negative force) from the tip after initially being attracted, was recorded against the conductivity of cell suspension medium

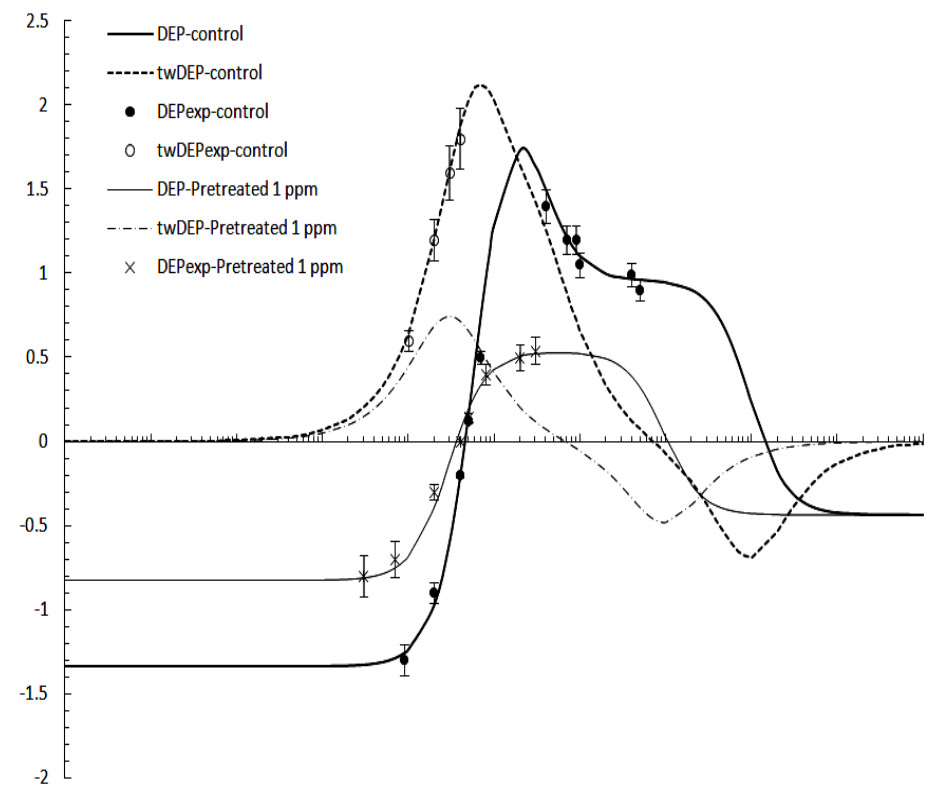

Figure 4. Experimental spectra of cells velocity for dielectrophoresis (DEPexp) and traveling wave dielectrophoresis (twDEPexp) were plotted as a function of the electric field frequency with theoretical curves of DEP and twDEP. 
$\left(\sigma_{s}\right)$. It was observed that as the $\sigma_{s}$ was increased the $f_{\ell}$ was shifted towards a higher frequency value for $S$. cerevisiae TISTR 5088, Tetraselmis sp. and HRBC. It should be noted that cell velocity spectra for all three cell types were reduced significantly at greater $\sigma_{\mathrm{s}}$ values. According to the model, when the increased $\sigma_{s}$ reached a critical value the attractive force became negligible, implying an equivalence to the cytoplasmic conductivity. Yeast cells at a cell density of $10^{5} \mathrm{ml}^{-1}$ displayed positive dielectrophoretic force over a frequency range between $50 \mathrm{kHz}$ and $15 \mathrm{MHz}$, when increasing $\sigma_{s}$ from 0.01 to 0.25 $\mathrm{S} . \mathrm{m}^{-1}$. For traveling wave dielectrophoresis, the cells experienced the traveling wave dielectrophoretic force at about 60 $\mathrm{kHz}$ and $\sigma_{s}$ of $0.01-0.25{\mathrm{~S} . \mathrm{m}^{-1}}^{-}$for electric field strength of $10-143 \mathrm{kV} / \mathrm{m}$. By curve-fitting methods to the model, it was shown that dielectric values of yeast were similar to what were reported using other methods $[11,15,16]$. The cytoplasmic and the membrane conductivity for yeast cells were $0.23 \mathrm{~S}_{\mathrm{m}} \mathrm{m}^{-1}$ and $0.1 \mu \mathrm{S} . \mathrm{m}^{-1}$, respectively. For Tetraselmis sp., cells experienced positive dielectrophoresis over a frequency ranged from $50 \mathrm{kHz}$ and $0.5 \mathrm{MHz}$, when increasing $\sigma_{\mathrm{s}}$ from 0.01 to $0.10 \mathrm{~S} . \mathrm{m}^{-1}$. Tetraselmis sp. experienced traveling wave dielectrophoresis in the 20-48 kHz frequency range with $\sigma_{s}$ of $0.01-0.37 ~ S . \mathrm{m}^{-1}$ for $28-143 \mathrm{kV} / \mathrm{m}$. In the case of HRBC, it was found that positive dielectrophoresis occurred in the range $8 \mathrm{kHz}-10 \mathrm{MHz}$, when increasing $\sigma_{s}$ from 0.64 to $60 \mathrm{mS} . \mathrm{m}^{-1}$. HRBC cells experienced traveling wave dielectrophoresis in the $50-150 \mathrm{kHz}$ frequency range with $\sigma_{s}$ of $56 \mathrm{~S}^{-1}$ and $4.7-14 \mathrm{kV} / \mathrm{m}$ for both type of A and B groups.

Dead yeast cells exhibited positive dielectrophoresis over the frequency range from $100 \mathrm{kHz}\left(f_{\ell}\right)$ to $1 \mathrm{MHz}$ if the medium conductivities were between 0.01-0.04 S.m ${ }^{-1}$. The value of $f_{\ell}$ was shifted towards greater values when $\sigma_{s}$ was increased. The spectra of cells translational velocity and critical frequency of the living and dead cells were different and allowed the different dielectric properties of these to be determined. The DEP velocity $\left(\vec{v}_{c D E P}\right.$ ) spectra and the value of $f_{\ell}$ of Tetraselmis sp. were investigated in control and arsenic treated cells. Increasing arsenic level from 1 to $150 \mathrm{ppm}$ reduced the magnitude of cell velocity and shifts the $f_{\ell}$ to a lower value. When the control and the arsenic contaminated cells were combined, one can distinguish the control from the arsenic pretreated cells if the frequency used was below $35 \mathrm{kHz}$.

The specific capacitance ( $\left.C_{m}\right)$ and conductance $\left(G_{m}\right)$ of the membrane were calculated for control and arsenic treated cells. The results showed that $C_{m}$ and $G_{m}$ of living and dead yeast cells were $10.1 \mathrm{mF} . \mathrm{m}^{-2}$, $8.3 \mathrm{~S} . \mathrm{m}^{-2}$ and $18.4 \mathrm{mF} . \mathrm{m}^{-2}, 33.3 \times 10^{3}$ S. $\mathrm{m}^{-2}$, respectively. Thus arsenic has a relatively small effect on $C_{m}$ but a very large effect on $G_{m}$ in yeast. The values of $C_{m}$ and $G_{m}$ of the control Tetraselmis sp. cells were $5.5 \mathrm{mF} . \mathrm{m}^{-2}$ and $13.0 \mathrm{~S} . \mathrm{m}^{-2}$, respectively, which were smaller than that of the arsenic pretreated cells. For 1, 5, 10, 50, and $150 \mathrm{ppm}$ pretreated cells, $C_{m}$ values were $6.7,8.3,10.8,14.3$ and 21.8 $\left(\mathrm{mF} . \mathrm{m}^{-2}\right)$, respectively. It was interesting that while the $C_{m}$ values of the arsenic pretreated cells were increased, the value of $G_{m}$ remained constant and the conductivity of cell membrane $\left(\sigma_{m}\right)$ was 23.0 S.m $\mathrm{m}^{-2}$.

\section{Acknowledgements}

This work has been supported by National Electronics and Computer Technology Center, National Science and Technology Development Agency (NSTDA), Thailand (grant NT-FD-B-22-EDS-19-54-05). Thanks are also extended to Vachira Phuket Hospital (Phuket, Thailand) and NICA for providing HRBCs and Tetraselmis sp. cells, respectively, and Dr. Raymond Ritchie for help with the manuscript.

\section{REFERENCES}

[1] S. Masuda, M. Washizu, and I. Kawabata, "Movement of blood cells by nonuniform traveling field,” IEEE Trans. Ind. Applicat., vol. 24, pp. 214-222, 1988.

[2] G. Fuhr, R. Hagedorn, T. Muller, W. Benecke, B. Wagner, and J. Gimsa, "Asynchronous traveling wave induced linear motion of living cells, " Journal of Studia Biophisica, vol. 140 (2), pp.79-102. 1991.

[3] R. Hagedorn, G. Fuhr, T. Muller, and J. Gimsa, "Traveling wave dielectrophoresis of microparticles," Electrophoresis, vol.13, pp.49-54, 1992.

[4] X. B.Wang, M. P. Hughes, Y. Huang, F. F. Becker, and P. R. C. Gascoyne, "Non-uniform spatial distributions of both the magnitude and phase of AC electric fields determine dielectrophoretic forces," Biochimica et Biophysica Acta., vol. 1243, pp.185-194, 1995.

[5] M. P. Hughes, "AC electrokinetic: applications for nanotechnology,” Nanotechnology, vol. 11, pp.124-132, 2000.

[6] T. B. Jones, "Basic theory of dielectrophoresis and electrorotation,” IEEE Engineering in Medicine and Biology Magazine, vol. 22(6), pp. 33-42, 2003.

[7] R. Pethig, M. S. Talary, and R. S. Lee, "Enhancing traveling-wave dielectrophoresis with signal superposition,” IEEE Engineering in Medicine and Biology Magazine, vol. 22(6), pp. 43-50, 2003.

[8] M. S.Talary, J. P. H. Burt, J. A. Tame, and R. J. Pethig, "Electromanipulation and separation of cells using traveling electric fields," Journal of Physics D: Applied Physics. Vol. 29, pp. 2198-2203, 1996.

[9] L. M. Fu, G.B. Lee, Y. H. Lin, and R. J. Yang, "Manipulation of microparticles using new modes of traveling wave dielectrophoretic force: Numerical simulation and experiments," Journal of IEEE/ASME Transactions (Mechatronics), vol. 9 (2), pp.377-383. 2004.

[10] E. G. Cen, C. Dalton, Y. Li, S. Adamia, L.M. Pilarski, and K.V.I.S. Kaler, “A combined dielectrophoresis, traveling wave dielectrophoresis and electrorotation microchip for the manipulation and characterization of human malignant cells," Journal of MicrobiologicalMethods, vol.58, pp.387-401, 2004.

[11] S. Bunthawin, P.Wanichapichart, A.Tuantranont, and H. G. L. Coster, "Dielectrophoretic spectra of translational velocity and critical frequency for a spheroid in traveling electric field,” Biomicrofluidics. 4:014102 [doi:10.1063/1.3294082], 2010.

[12] L. D. Landau and E. M. Liftschitz, Elektrodanamik der kontinua. Akademie Verlag, Berlin, 1985.

[13] K. Asami, "Characterization of biological cells by dielectric spectroscopy,”Non-Crystal Solids, vol. 305, pp. 268-277, 2002.

[14] P. Wanichapichart, T. Wongluksanapan, and L. Khooburat, "Electrorotation: diagnostic tool for abnormality of marine phytoplankton cells," Proceedings of the 2nd IEEE International Conference on Nano/Micro Engineering and Molecular Systems, 
Bangkok, Thailand, January 16 - 19, pp.1115-1120, 2007.

[15] X. F. Zhou, G. H. Markx, and R. Pethig, "Effect of biocide concentration on electrorotation spectra of yeast cells," Biochim. Biophys. Acta., vol.1281, pp. 60-64, 1996.
[16] S. Bunthawin, P.Wanichapichart, and J.Gimsa, "An investigation of dielectric properties of biological cells using RC-model," Songklanakarin Journal of Science and Technology, vol.29 (4), pp.1163-1181, 2007. 